\title{
Neurocysticercosis in Nepal: a retrospective clinical analysis
}

\author{
Rajeev Ojha ${ }^{1}$, Dinesh Bikram Shah ${ }^{1}$, Amina Shrestha ${ }^{1}$, Sunil Koirala ${ }^{1}$, Apurba Dahal ${ }^{2}$, Khem Adhikari ${ }^{1}$, \\ Anjal Bisht ${ }^{1}$, Pratik Wagle ${ }^{1}$ \\ ${ }^{1}$ Department of Medicine, Neurology Unit, National Academy of Medical Sciences, Bir Hospital, Mahaboudha, Kathmandu 44600, Nepal. \\ ${ }^{2}$ Department of Medicine, Om Hospital and Research Center, Chabahil, Kathmandu 44602, Nepal.
}

\section{A B S T R A C T}

\begin{abstract}
Aim: The prevalence of epilepsy is higher in Nepal. This study was conducted to analyze the clinical manifestations of neurocysticercosis (NCC) among seizure patients admitted to our center. Methods: We retrospectively studied all the NCC patients admitted to Neurology Department, Bir Hospital, Kathmandu, Nepal from April 2012 to February 2014. Computer tomography/ magnetic resonance imaging $(\mathrm{CT} / \mathrm{MRI})$ head, clinical profile, lab investigations and exclusion of other causes were the basis of the NCC diagnosis. Chi-square and Student's $t$-test were used for comparison of variables. Results: Out of 131 seizure patients admitted, 21 patients were diagnosed with NCC [mean age: $33.95 \pm 16.41$; male: $15(71.4 \%)$, female: $6(28.6 \%)$ ]. Generalized tonic clonic seizure was the most common seizure type in NCC patients (18 patients; $85.7 \%$ ), two of them had status epilepticus during presentation in Emergency Department. Three patients had focal seizure, one with epilepsia partialis continua. Neuroimaging showed multiple NCC lesions in 8 (38.1\%) and a single NCC lesion in $13(61.9 \%)$ patients. Seven of them (33.3\%) sought traditional healers before being presented to our center. Eight patients (38.1\%) were treated with antiepileptics in local health-post without neuroimaging studies done. Calcified stage of NCC was the most frequent CT/MRI findings (12 patients; $57.1 \%$ ). Phenytoin was preferred both by physicians and patients due to its low cost. Conclusion: NCC is a common finding among seizure patients in Nepal. Poor economic status, illiteracy and underdeveloped rural society are the major challenges in prevention and treatment of NCC.
\end{abstract}

Key words: Cysticercosis, epilepsy, neurocysticercosis, Nepal, south Asia

\section{INTRODUCTION}

Neurocysticercosis (NCC) is the most common parasitic infection of the human brain. It is caused by the larval stage of Taenia solium, which enters the central nervous system by ingestion of its eggs due to the use of contaminated hand, water or food. ${ }^{[1]}$ Being a low-income country, the majority of Nepalese population is still illiterate and deal with crops and animal farming. Due to lack of toilets in many Nepalese homes, open field defecation is a common practice. Thus, poor sanitation and improper management of food and meat products are the major causes for higher NCC cases in Nepal.

Seizure is the most common clinical manifestation of NCC, followed by focal neurological deficits and

\begin{tabular}{|c|c|}
\hline \multicolumn{2}{|c|}{ Access this article online } \\
\hline Quick Response Code: & \multirow[b]{2}{*}{$\begin{array}{l}\text { Website: } \\
\text { www.nnjournal.net }\end{array}$} \\
\hline & \\
\hline & $\begin{array}{l}\text { DOI: } \\
10.4103 / 2347-8659.160856\end{array}$ \\
\hline
\end{tabular}

headache. Diagnosis is often difficult even in developed and non endemic areas, where NCC lesions are confused with tuberculoma or metastatic lesions. ${ }^{[2,3]}$ Due to unavailability of laboratory tests and radioimaging tools, diagnosis of NCC is often challenging in developing countries. A large treatment gap has been reported from developing countries, $70 \%$ in Nepal, ${ }^{[4]}$ $96 \%$ in Nigeria, ${ }^{[5]} 73 \%$ in Pakistan ${ }^{[6]}$ and $90 \%$ in India. ${ }^{[7]}$ Among these, epidemiological studies reported higher prevalence of the treatment gap in rural areas in India and Pakistan. ${ }^{[6,7]}$

Since only a few NCC reports were conducted in Nepal, this study aims at describing the clinical manifestations of NCC among seizure patients admitted to our center.

\section{METHODS}

We retrospectively analyzed all the available data of NCC patients who were admitted to Neurology Department, Bir Hospital, Kathmandu, Nepal from April 2012 to February 2014. Computer tomography and magnetic resonance imaging (CT and MRI) head were used to classify the various stages of NCC. ${ }^{[8]}$

Corresponding Author: Dr. Rajeev Ojha, Department of Medicine, Neurology Unit, National Academy of Medical Sciences, Bir Hospital, Mahaboudha, Kathmandu 44600, Nepal. E-mail: rajeevnet@hotmail.com 
CT/MRI head, clinical profile, laboratory investigations and exclusion of other causes were the basis of NCC diagnosis. Fundus examination was done in all patients to rule out ocular NCC. Out of 21 NCC patients, MRI Brain was available for 2 patients and CT head plain, and contrast were performed on the remaining patients (19). Serum, anticysticercal antibodies test, was carried out in 2 patients, and serum titer $(>1: 160)$ was positive in one of them. NCC was diagnosed according to Del Brutto ${ }^{[9]}$ revised diagnostic criteria for NCC. These include absolute, major, minor and epidemiological criteria, and the degrees of diagnostic certainty can be further classified under definitive and probable diagnosis. Reports of electroencephalography, complete blood count, chest X-ray, Mantoux test, sputum for acid fast bacilli, renal and liver function test, abdominal ultrasonography and electrocardiography were collected from each patient. The institution ethical board approved the study protocol.

Patients with an intracranial malignancy, head injury, stroke, metabolic disturbance, tuberculosis, brain abscess and HIV were excluded. Patients of pediatric age, that is, below 15 years were not included in our study. Details of patient's personal data, socioeconomic status, clinical profile, drug history and response were recorded.

Data storage and statistical analysis were performed by IBM SPSS Statistics Version 20 for Mac (IBM Corporation, New York, United States). Chi-square and Student's $t$-test were used for comparison of variables; a two-tailed $P<0.05$ was considered statistically significant.

\section{RESULTS}

Out of 131 seizure patients, $46.5 \%$ were diagnosed with alcohol withdrawal seizure, followed by idiopathic generalized seizure $(17.5 \%)$, NCC $(16.0 \%)$ and others $20 \%$. NCC was the most common cause of secondary epilepsy, followed by brain tuberculoma (3.8\%), stroke $(3.8 \%)$, encephalitis $(3.0 \%)$, and brain abscess (3.0\%) [Table 1].

Neurocysticercosis was diagnosed in 21 patients (16.0\%) with mean age of $33.9 \pm 16.4$ and male:female ratio was 15:6 [Table 2]. According to of the NCC criteria described by Del Brutto, ${ }^{[9]} 10$ patients were classified under definitive diagnosis (1 absolute or combination or major, minor and epidemiologic criteria) and 11 patients were diagnosed as probable NCC (combination of major, minor and epidemiologic criteria). About $30 \%$ of our NCC patients were local people of Kathmandu. Neuroimaging showed multiple NCC lesions in $8(38.1 \%)$ and a single NCC lesion in 13 (61.9\%). Generalized tonic clonic seizure was the

\begin{tabular}{|c|c|c|c|c|}
\hline & $\begin{array}{l}\text { Total } \\
n(\%)\end{array}$ & $\begin{array}{l}\text { Male } \\
n(\%)\end{array}$ & $\begin{array}{c}\text { Female } \\
n(\%)\end{array}$ & $P$ \\
\hline $\begin{array}{l}\text { Alcohal withdrawal } \\
\text { syndrome }\end{array}$ & $61(46.5)$ & $58(59.8)$ & $3(8.8)$ & $<0.001^{b}$ \\
\hline $\begin{array}{l}\text { Idiopathic } \\
\text { generalized seizure }\end{array}$ & $23(17.5)$ & $8(8.2)$ & $15(44.1)$ & $<0.001^{\mathrm{b}}$ \\
\hline NCC & $21(16.0)$ & 15 (15.5) & $6(17.6)$ & 0.765 \\
\hline Encephalitis & $4(3.0)$ & $3(3.1)$ & $1(2.9)$ & 1.0 \\
\hline Brain abscess & $4(3.0)$ & $1(1.0)$ & $3(8.8)$ & 0.053 \\
\hline \multicolumn{5}{|l|}{ Stroke } \\
\hline Hemorrhagic & $3(2.3)$ & $1(1.0)$ & $2(5.9)$ & 0.165 \\
\hline Ischemic & $2(1.5)$ & 0 & $2(5.9)$ & a \\
\hline Tuberculoma & $5(3.8)$ & $3(3.1)$ & $2(5.9)$ & 0.604 \\
\hline Others & $8(6.1)$ & $5(5.1)$ & $3(8.8)$ & 0.427 \\
\hline Total & 131 & 97 & 34 & \\
\hline
\end{tabular}

Data are shown as $n(\%)$ or mean \pm SD. ${ }^{a} P$ value not applicable; ${ }^{b} P<0.001$. SD: standard deviation; NCC: neurocysticercosis

\begin{tabular}{lcccc}
\hline \multicolumn{5}{c}{ Table 2: Characteristics of NCC lesions } \\
\hline & Total $\boldsymbol{n}(\%)$ & Male $\boldsymbol{n}(\%)$ & Female $\boldsymbol{n}(\%)$ & $\boldsymbol{P}$ \\
\hline $\begin{array}{l}\text { Age (years) } \\
\text { Lesion }\end{array}$ & $33.9 \pm 16.4$ & $33.0 \pm 16.6$ & $36.3 \pm 17.1$ & 0.685 \\
$\quad$ & & & & \\
$\quad$ Single & $13(61.9)$ & $9(60.0)$ & $4(66.7)$ & 1.0 \\
$\quad$ Multiple & $8(38.1)$ & $6(40.0)$ & $2(33.3)$ & \\
Seizure & & & & \\
$\quad$ GTCS & $18(85.7)$ & $14(93.3)$ & $4(66.7)$ & 0.184 \\
$\quad$ Partial & $3(14.3)$ & $1(6.7)$ & $2(33.3)$ & \\
CT/MRI stage & & & & \\
Multiple & $3(14.3)$ & $2(13.3)$ & $1(16.7)$ & 1.0 \\
I & 0 & 0 & 0 & a \\
II & $5(23.8)$ & $4(26.7)$ & $1(16.7)$ & 1.0 \\
III & $1(4.8)$ & $1(6.7)$ & 0 & $a$ \\
IV & $12(57.1)$ & $8(53.3)$ & $4(66.7)$ & 0.659 \\
\hline
\end{tabular}

Data are shown as $n(\%)$ or mean \pm SD. a $P$ value not applicable. GTCS: generalized tonic clonic seizure; $\mathrm{CT}$ : computer tomography; MRI: magnetic resonance imaging; SD: standard deviation; NCC: neurocysticercosis

most common seizure type in NCC patients (18 patients; 85.7\%), two of whom had status epilepticus during presentation in an emergency department. Three patients (14.3\%) had focal seizure, one of them had epilepsia partialis continua. Headache and vomiting were observed in 7 (33.3\%) and $5(23.8 \%)$ patients, respectively [Figure 1]. Calcified stage of NCC was the most frequent CT/MRI findings (12 patients; $57.1 \%$ ).

Seven patients (33.3\%) had sought traditional healers before they were referred to our center. Eight patients (38.1\%) were found to be treated with antiepileptics in local health-post without neuroimaging studies done. Phenytoin was preferred both by physicians and patients due to its low cost (12 patients; 57.1\%). Valproic acid (5 patients; $23.8 \%$ ) and carbamazepine (4 patients, $19.04 \%)$ were other common first generation antiepileptic drugs (AEDs) reported in our study. Multiple AEDs were administered during treatment of 2 patients.

\section{DISCUSSION}

Neurocysticercosis is an endemic disease and a health burden in Nepal. In our study, it is the most common 


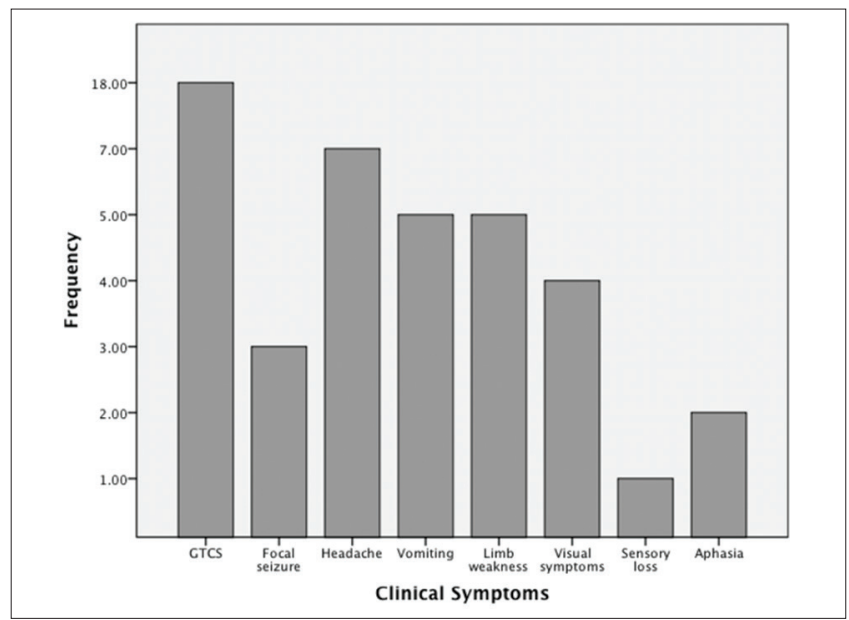

Figure 1: Clinical manifestations in neurocysticercosis patients

acquired cause of the seizure. Low socioeconomic status, poor hygiene and improper management in pigs rearing are the causes of high NCC prevalence in Nepal. ${ }^{[10]}$ Due to migration from rural areas, NCC is recently increasing in its prevalence also in urban areas. In our study, about $30 \%$ of the patients were from Kathmandu valley. Recently, increasing NCC prevalence in nonendemic or developed countries is due to migrant workers and international travellers. ${ }^{[11]}$ Few studies from China, ${ }^{[12]}$ Brazil $^{[13,14]}$ and India ${ }^{[15]}$ also have shown the higher NCC cases reported in urban areas.

Our study shows NCC is common among young adults. Male patients are more common than female among NCC patients. Female predominance was seen in some studies. ${ }^{[16,17]}$ Single NCC lesions are found to be more common than multiple NCC lesions [Table 2]. Similar results were reported from various previous studies. ${ }^{[18-20]}$ However, a pediatric study from Nepal reported higher prevalence of multiple lesions among NCC patients. ${ }^{[21]}$ No extraparenchymal lesions was observed in our study, in contrast with what reported by Basu et al. ${ }^{[21]}$ showing 20\% of extraparenchymal lesions.

Calcified granuloma may act as a seizure focus and may lead to epilepsy. ${ }^{[22]}$ Calcified lesion with perilesional edema was the most common NCC stage among our patients. About 23.8\% patients were classified under colloidal stage, which consisted of active inflammation of the lesion along with scolex degeneration. ${ }^{[23]}$ Since vesicular stage is usually asymptomatic due to its least inflammatory response to the surrounding brain tissues. Meanwhile, this stage is commonly found to be associated with lesions of other stages, being 14.3\% in our study. ${ }^{[1]}$

Seizure is the most common presentation in NCC, ranges from $60 \%$ to $90 \% .{ }^{[16,18]}$ Almost all NCC patients were presented with seizure, and most of them had focal neurological deficits (limb weakness, visual disturbance, aphasia, etc.). This might be attributed to the fact that only the severe and disabling patient visited our hospital. Furthermore, it is a popular in Nepalese practice that most seizure patients initially visit traditional healers before presenting to hospital. ${ }^{[4]}$ Similar to the study of Bhattacharjee et al., ${ }^{[18]}$ the frequency of status epilepticus was low in our patient cohort. Consistent with our observations, generalized seizure was common in some other previous studies. ${ }^{[20,24]}$ However, most of them reported focal seizure as the major seizure type. ${ }^{[15,25,26]}$ A Nepalese pediatric study has also reported focal seizure in about $30 \%$ of their patients. ${ }^{[21]}$

Visiting traditional healers is popular among Nepalese population, especially in remote areas. ${ }^{[4]}$ Furthermore, lack of personal health, low socioeconomic status, and imaging techniques are the challenges for diagnosis of NCC.

In conclusion, neurocysticercosis is a major cause of acquired epilepsy in Nepal. Low economic status, illiteracy, and underdeveloped rural society are the major obstacles to prevention and treatment of NCC. Due to economic factors and inaccessible AEDs in remote places in Nepal, discontinuation of the drug is common. Proper management of NCC is needed to minimize the prevalence of epilepsy in developing countries.

This is a single center study, and we were unable to recruit the desired number of patients. Due to unavailability and poor economic condition of most of the patients, serum anticysticercal antibodies test was performed in 2 patients only.

\section{ACKNOWLEDGMENTS}

We acknowledge with gratitude the efforts of record keeping department and nursing staffs of Neurology Unit, Bir Hospital.

\section{REFERENCES}

1. Nash TE, Garcia HH. Diagnosis and treatment of neurocysticercosis. Nat Rev Neurol 2011;7:584-94.

2. Agarwal JP. Neurocysticercosis in Nepal and it's global perspective. Kathmandu Univ Med J (KUMJ) 2011;9:1-2.

3. Salzer HJ, Hoenigl M, Valentin T, Zollner-Schwetz I, Krause R. Neurocysticercosis in a European traveler returning from India primarily suspected as neoplasia. J Travel Med 2013;20:333-5.

4. Rajbhandari KC. Epilepsy in Nepal. Can JNeurol Sci 2004;31:257-60.

5. Osuntokun BO, Adeuja AO, Nottidge VA, Bademosi O, Olumide A, Ige O, Yaria F, Bolis CL, Schoenberg BS. Prevalence of the epilepsies in Nigerian Africans: a community-based study. Epilepsia 1987;28:272-9.

6. Aziz H, Ali SM, Frances P, Khan MI, Hasan KZ. Epilepsy in Pakistan a population-based epidemiologic study. Epilepsia 1994;35:950-8.

7. Bharucha NE. Epidemiology and treatment gap of epilepsy in India. Ann Indian Acad Neurol 2012;15:352-3. 
8. Coyle CM, Tanowitz HB. Diagnosis and treatment of neurocysticercosis. Interdiscip Perspect Infect Dis 2009;2009:180742.

9. Del Brutto OH. Diagnostic criteria for neurocysticercosis, revisited. Pathog Glob Health 2012;106:299-304.

10. Rajshekhar V, Joshi DD, Doanh NQ, van De N, Xiaonong Z. Taenia solium taeniosis/cysticercosis in Asia: epidemiology, impact and issues. Acta Trop 2003;87:53-60.

11. Del Brutto OH, García HH. Neurocysticercosis in nonendemic countries: time for a reappraisal. Neuroepidemiology 2012;39:145-6.

12. Chaoshuang L, Zhixin Z, Xiaohong W, Zhanlian H, Zhiliang G. Clinical analysis of 52 cases of neurocysticercosis. Trop Doct 2008;38:192-4

13. das Chagas Md, D'Oliveira Júnior A, Tavares-Neto J. Clinical aspects of neurocysticercosis at semi-desert region of Brazilian northeast. Arq Neuropsiquiatr 2003;61:398-402.

14. Benedeti MR, Falavigna DL, Falavigna-Guilherme AL, Araújo SM. Epidemiological and clinical profile of neurocysticercosis patients assisted by the Hospital Universitário Regional de Maringá, Paraná, Brazil. Arq Neuropsiquiatr 2007;65:124-9.

15. Rajshekhar V, Raghava MV, Prabhakaran V, Oommen A, Muliyil J. Active epilepsy as an index of burden of neurocysticercosis in Vellore district, India. Neurology 2006;67:2135-9.

16. Singhi P, Ray M, Singhi S, Khandelwal N. Clinical spectrum of 500 children with neurocysticercosis and response to albendazole therapy. J Child Neurol 2000;15:207-13.

17. Morgado C, Gomes LB, de Campos JG. Neurocysticercosis. An imaging analysis of 35 cases. Acta Med Port 1994;7:269-75.

18. Bhattacharjee S, Biswas P, Mondal T. Clinical profile and follow-up of 51 pediatric neurocysticercosis cases: a study from Eastern India. Ann Indian Acad Neurol 2013;16:549-55.

19. Singhi P, Singhi S. Neurocysticercosis in children. J Child Neurol
2004; 19:482-92

20. Gauchan E, Malla T, Basnet S, Rao KS. Variability of presentations and CT-scan findings in children with neurocysticercosis. Kathmandu Univ Med J (KUMJ) 2011;9:17-21.

21. Basu S, Ramchandran U, Thapliya A. Clinical profile and outcome of pediatric neurocysticercosis: a study from Western Nepal. J Pediatr Neurol 2007;1:45-52.

22. Nash T. Edema surrounding calcified intracranial cysticerci: clinical manifestations, natural history, and treatment. Pathog Glob Health 2012;106:275-9.

23. Lucato LT, Guedes MS, Sato JR, Bacheschi LA, Machado LR, Leite CC. The role of conventional MR imaging sequences in the evaluation of neurocysticercosis: impact on characterization of the scolex and lesion burden. AJNR Am J Neuroradiol 2007;28:1501-4.

24. Del Brutto OH, Santibañez R, Noboa CA, Aguirre R, Díaz E, Alarcón TA. Epilepsy due to neurocysticercosis: analysis of 203 patients. Neurology 1992;42:389-92.

25. Morales NM, Agapejev S, Morales RR, Padula NA, Lima MM. Clinical aspects of neurocysticercosis in children. Pediatr Neurol 2000;22:287-91.

26. Talukdar B, Saxena A, Popli VK, Choudhury V. Neurocysticercosis in children: clinical characteristics and outcome. Ann Trop Paediatr 2002;22:333-9.

Cite this article as: Ojha R, Shah DB, Shrestha A, Koirala S, Dahal A, Adhikari K, Bisht A, Wagle P. Neurocysticercosis in Nepal: a retrospective clinical analysis. Neuroimmunol Neuroinflammation 2015;2(3):167-70.

Source of Support: Nil. Conflict of Interest: No.

Received: 12-11-2014; Accepted: 19-02-2015 